\title{
On the possibility of decoherence due to relativistic effect
}

\author{
B. Carazza \\ Dipartimento di Fisica dell' Università, \\ viale delle Scienze, I43100 Parma, Italy \\ INFN Sezione di Cagliari, Italy
}

\begin{abstract}
This note looks at the possibility of a system of free particles presenting decoherence in the total momentum when tracing upon their relative momenta if we take into account a relativistic correction to the expression of the kinetic energy.
\end{abstract}

Key words: Decoherence, reduced density matrix, preferred basis, isolated system.

PACS: 03.65.Bz 


\section{INTRODUCTION}

Decoherence - the phenomenon whereby a quantum system initially in a pure state evolves into a improper mixture due to interaction with its environment [1] - has been studied extensively, using both toy models 20 and more complex models representing real physical situations 3]. Sources of decoherence such as scattering [4, 5] and quantum gravity [6, 7] and both internal [8, 9] and external[10, 11] environment are taken into consideration. Omnes 12 recently presented a general theory of the effect of decoherence that includes both the harmonic model of Caldeira-Legett [10] and the external environment considered by Joos and Zeh[11] and is related to the quantum state[13 diffusion model.

Decoherence is especially interesting as it promise to solve the old problem of how to derive the classical behaviour of macroscopic bodies from quantum principles. In fact, in the case of a macroscopic body, decoherence is believed to suppress the off-diagonal elements of the spatial reduced matrix of the centre of mass, irrespective of initial conditions. This is equivalent to establishing a superselection rule for the position of macroscopic bodies and to saying that we cannot experience spatial macrosuperpositions. We can thus explain why we do not encounter states of this kind when looking at everyday objects. To derive such a result it is not necessary to consider an external environment. In fact, a system of many particles like a macroscopic body may be considered as consisting formally of a "collective system" described by the collective coordinates, and the system described by its microscopic coordinates, which can act as an (internal) environment if the two formal systems are coupled. This coupling may be either guaranteed by some constraints or caused by an external potential. Of course in the case of an isolated macroscopic body, the Hamiltonian consist of the sum of two separate Hamiltonians relative to the centre of mass and internal variables respectively, there is no coupling between them and so decoherence of the collective variable is not possible. However, we wish to signal a possible case of decoherence, with suppression of correlations in the total momentum, for a system of particles even if isolated, provided that we consider relativistic

corrections to the non-relativistic Hamiltonian. It would pointed out that de- 
coherence of isolated systems has been discussed before in various contexts: Halliwell[8] investigated the variables that will generally become effectively classical as the local densities (of number of particles, momentum, energy), Calzetta and $\mathrm{Hu}$ 14 the decoherence of histories of certain correlation functions in a field theory. We should also mention that the diagonalization in the square of momentum basis has been argued for a system of (non-relativistic) particles interacting with the linearized gravitational field 15.

It is know that in classical electrodynamic a system of interacting particles may be approximately described to terms of second order in $1 / c$ by a Hamiltonian depending only on their positions and velocities, since the radiation comes in with the terms of order $1 / c^{3}$. The same may be said for particles subject to gravitational interaction 16]. In this approximation the total momentum of the system is no longer independent, but is coupled to the internal degrees of freedom. So, if we adopt the above mentioned Hamiltonian (after due symmetrization) as the Hamiltonian operator within non-relativistic quantum mechanics, we may expect to have decoherence for the collective variable tracing over the microscopic ones. This may occurs even if the particles are not interacting, thanks to the relativistic correction to their kinetic energy. The coupling we are talking about commute with the non-relativistic free Hamiltonian of the centre of mass (c.m.). As we learned from studies of wide-open quantum systems [17], the diagonalization is to be expected in the basis of any dynamical operator commuting with the coupling terms and so your candidate to be a preferred basis is that of total momentum. The importance of an interaction commuting with the preferred basis was already stressed by Zurek[18].

Since the aim of this note is merely to briefly signal such a possibility, we will consider for the sake of simplicity a system of $n$ free spinless particles of equal mass $m$ in one dimension, a one dimensional ideal gas in fact. We stress that we intend to work within the non-relativistic quantum mechanics and consider the correction to the kinetic energy and its consequences as a relativistic effect.

The model certainly is not realistic but we intend to offer the result as a further case of decoherence with not usual features. Not usual because the system is isolated and also because in our case the reduced statistical operator diagonalizes on the basis of the total momentum, which thus acquires the status of preferred basis. We believe all this is interesting in itself. We do like to stress that the calculations will be carried out without recourse to 
any master equation but by following the Schroedinger evolution of a pure state. With the kinetic operator used, the Galilean invariance is destroyed, so we have to specify the reference frame we are referring to. A Galilean transformation corresponds to a translation of momenta. For the approximation we are using to make any sense, the reference frames to take into consideration are obviously the ones (if they exist) for which the absolute value of momentum for each particle is not significantly likely to be of the order of or greater than $m c$. This requisite can be expressed by applying the condition $\left\langle\widehat{p}_{i}^{2}\right\rangle \ll m^{2} c^{2}$ for every $i$. It will certainly be satisfied if the more restrictive condition $\sum_{i=1}^{n}\left\langle\widehat{p}_{i}^{2}\right\rangle \ll m^{2} c^{2}$ also is. To establish a definite reference frame, we will adopt the one in which, for a given wave function, the last mentioned expression gives the lowest value. It will be the reference frame in which the mean value of total momentum is zero. Even selecting the optimal reference frame, however, the validity of our approximation is not automatically guaranteed and our result will be only significant if the condition on the values of $\left\langle\widehat{p}_{i}^{2}\right\rangle$ is satisfied.

\section{THE SYSTEM OF FREE PARTICLES IN ONE DIMENSION WITH RELATIVISTIC CORRECTION TO THE KINETIC EN- ERGY}

The free Hamiltonian which we will consider for quasi-relativistic particles is:

$$
\sum_{i=1}^{n} \frac{\widehat{p}_{i}^{2}}{2 m}-\sum_{i=1}^{n} \frac{\widehat{p}_{i}^{4}}{8 m^{3} c^{2}} \quad .
$$

Using the momentum basis and with $\varphi\left(p_{1}, p_{2}, \ldots, p_{n}\right)$ indicating the initial state, we have the state at time $t$ as follows:

$$
|\chi(t)\rangle=e^{-i t / \hbar\left[\sum_{i=1}^{n}\left(\frac{p_{i}^{2}}{2 m}-\frac{p_{i}^{4}}{8 m^{3} c^{2}}\right)\right]} \varphi\left(p_{1}, p_{2}, \ldots, p_{n}\right) \quad .
$$

We now change the variables: $p_{i}=P / n+\eta_{i}$, where $P$ is the total momentum. The $\eta_{i}$ are not independent, since they must satisfy the relation $\sum_{i=1}^{n} \eta_{i}=0$. We will consider as independent variables $P$ together with the first $n-1$ relative momenta. The last relative momentum is expressed as $\eta_{n}=-\sum_{i=1}^{n-1} \eta_{i}$. The Jacobian determinant of the transformation is equal to 1 . We will write the integration volume element for the new variables as $d V=d P d S$, where 
$d S=d \eta_{1} d \eta_{2} \cdots d \eta_{n-1}$. Using the new variables and taking into account the relation $\sum_{i=1}^{n} \eta_{i}=0$ the expression above now becomes:

$$
|\chi(t)\rangle=e^{-\frac{i t}{\hbar}\left[\frac{P^{2}}{2 n m}-\frac{P^{4}}{8 n^{3} m^{3} c^{2}}+\sum_{i=1}^{n}\left(\frac{\eta_{i}^{2}}{2 m}-\frac{\eta_{i}^{4}}{8 m^{3} c^{2}}-\frac{3 P^{2}}{4 n^{2} m^{3} c^{2}} \eta_{i}^{2}-\frac{P}{2 n m^{3} c^{2}} \eta_{i}^{3}\right)\right]} \Phi\left(P, \eta_{1}, \ldots, \eta_{n}\right)
$$

where $\Phi\left(P, \eta_{1}, \eta_{2}, \ldots, \eta_{n}\right)$ indicates $\varphi\left(P / n+\eta_{1}, P / n+\eta_{2}, \ldots, P / n+\eta_{n}\right)$ Note that here and in the various expressions that follow $\eta_{n}$ stands for $=-\sum_{i=1}^{n-1} \eta_{i}$. The projection $\left\langle P^{\prime} \mid \chi(t)\right\rangle$ of the state vector at time $t$ on an eigenstate $\left|P^{\prime}\right\rangle$ of the total momentum is:

$$
e^{-\frac{i t}{\hbar}\left[\frac{P^{\prime 2}}{2 n m}-\frac{P^{\prime 4}}{8 n^{3} m^{3} c^{2}}+\sum_{i=1}^{n}\left(\frac{\eta_{i}^{2}}{2 m}-\frac{\eta_{i}^{4}}{8 m^{3} c^{2}}-\frac{3 P^{\prime 2}}{4 n^{2} m^{3} c^{2}} \eta_{i}^{2}-\frac{P^{\prime}}{2 n m^{3} c^{2}} \eta_{i}^{3}\right)\right]} \Phi\left(P^{\prime}, \eta_{1}, \ldots, \eta_{n}\right) .
$$

By tracing $\left\langle\chi(t) \mid P^{\prime \prime}\right\rangle\left\langle P^{\prime} \mid \chi(t)\right\rangle$ upon the relative momenta i.e. integrating over $d S$ we get the reduced density matrix element on the total momentum basis:

$$
\rho_{P^{\prime} P^{\prime \prime}}=e^{\frac{-i t}{\hbar}\left[\frac{\left(P^{\prime 2}-P^{\prime \prime 2}\right)}{2 n m}-\frac{\left(P^{\prime 4}-P^{\prime \prime 4}\right)}{64 m^{3} c^{2}}\right]} I_{P^{\prime} P^{\prime \prime}} \quad .
$$

The factor $I_{P^{\prime} P^{\prime \prime}}$ indicates the integral

$$
I_{P^{\prime} P^{\prime \prime}}=\int \Phi\left(P^{\prime}, \eta_{1}, \eta_{2}, \ldots, \eta_{n}\right) \Phi^{*}\left(P^{\prime \prime}, \eta_{1}, \eta_{2}, \ldots, \eta_{n}\right) e^{i t \sum_{i=1}^{n}\left(w \eta_{i}^{2}+z \eta_{i}^{3}\right)} d S
$$

where $w=3\left(P^{\prime 2}-P^{\prime \prime 2}\right) /\left(4 \hbar n^{2} m^{3} c^{2}\right)$ and $z=\left(P^{\prime}-P^{\prime \prime}\right) /\left(2 \hbar n m^{3} c^{2}\right)$. The diagonal matrix elements are constant of motion, but when $P^{\prime} \neq P^{\prime \prime}$ the integral in the previous expression may cancel out with time, in which case the off-diagonal elements are cancelled out, meaning that we have decoherence.

\section{EVALUATION OF THE REDUCED DENSITY MATRIX EL- EMENTS}

In order to understand the time behaviour of the matrix elements we are interested in is thus necessary to evaluate the integral $I_{P^{\prime} P^{\prime \prime}}$. To take into account the constraint $\eta_{n}=-\sum_{i=1}^{n-1} \eta_{i}$ we multiply the integrand by the delta function $\delta\left(\sum_{i=1}^{n} \eta_{i}\right)$ using its Fourier representation $(1 / 2 \pi) \int e^{i \sum_{i=1}^{n} k \eta_{i}} d k$ and consider $\eta_{n}$ too as a independent integration variable. Writing

$$
G\left(P^{\prime}, P^{\prime \prime} ; \eta_{1}, \ldots, \eta_{n}\right)=\Phi\left(P^{\prime}, \eta_{1}, \eta_{2}, \ldots, \eta_{n}\right) \Phi^{*}\left(P^{\prime \prime}, \eta_{1}, \eta_{2}, \ldots, \eta_{n}\right)
$$


and $h\left(\eta_{i}\right)=w \eta_{i}^{2}+z \eta_{i}^{3}$ our integral becomes

$$
\begin{aligned}
I_{P^{\prime} P^{\prime \prime}}= & (1 / 2 \pi) \int d k \int e^{i k \eta_{1}+i t h\left(\eta_{1}\right)} d \eta_{1} \int e^{i k \eta_{2}+i t h\left(\eta_{2}\right)} d \eta_{2} \ldots \\
& \int e^{i k \eta_{n}+i t h\left(\eta_{n}\right)} d \eta_{n} G\left(P^{\prime}, P^{\prime \prime} ; \eta_{1}, \eta_{2}, \ldots, \eta_{n}\right)
\end{aligned}
$$

Let us start with the integration on $\eta_{n}$. We are interested in the behaviour of the off diagonal matrix elements at large time, so we shall use asymptotic expansions methods to evaluate the result. More precisely, we will look at the method of stationary phase 19. According to which, when $t$ is a large quantity, the major contribution to the value of the integral arises from the immediate vicinity of the end points and from the vicinity of those point at which $h\left(\eta_{n}\right)$ is stationary, i.e. its first derivative $h^{\prime}\left(\eta_{n}\right)$ is equal to zero. In the first approximation the contribution of stationary points is more important. If we suppose that the function representing the intial state is square integrable and thus going to zero at infinity, we can disregard the end points contribution at all. Since only the neighbourhood matters, asymptotic evaluation consists of replacing $h\left(\eta_{n}\right)$ near any stationary point $\eta_{n}^{*}$ as $h\left(\eta_{n}^{*}\right)+(1 / 2) h^{\prime \prime}\left(\eta_{n}^{*}\right)\left(\eta_{n}-\eta_{n}^{*}\right)^{2}$. By the same argument, the factor of $e^{i t h\left(\eta_{n}\right)}$ in the integrand, assuming it is a continuous function, is replaced by its value at $\eta_{n}^{*}$ and the integration is safely extended from $-\infty$ to $+\infty$. We will replace $G\left(P^{\prime}, P^{\prime \prime} ; \eta_{1}, \ldots, \eta_{n}\right)$ by $G\left(P^{\prime}, P^{\prime \prime} ; \eta_{1}, \ldots, \eta_{n}^{*}\right)$, but maintain the dependence on $\eta_{n}$ of $e^{i k \eta_{n}}$, since it belongs to the Fourier representation of the delta function, a non-continuous and singular function indeed. We have two stationary points at $\eta_{n}=0$ and $\eta_{n}=-2 w /(3 z)=-\left(P^{\prime}+P^{\prime \prime}\right) / 2$ These two values are indicated as $\alpha_{1}$ and $\alpha_{2}$ respectively. The corresponding values of the second derivative are $h^{\prime \prime}\left(\alpha_{1}\right)=2 w$ and $h^{\prime \prime}\left(\alpha_{2}\right)=-2 w$. Using the procedure illustrated above, we obtains:

$$
\begin{gathered}
G\left(P^{\prime}, P^{\prime \prime} ; \eta_{1}, \eta_{2}, \ldots, \alpha_{1}\right)\left[2 i \pi / t h^{\prime \prime}\left(\alpha_{1}\right)\right]^{1 / 2} e^{i t h\left(\alpha_{1}\right)+i k \alpha_{1}-i k^{2} /\left(2 t h^{\prime \prime}\left(\alpha_{1}\right)\right)}+ \\
G\left(P^{\prime}, P^{\prime \prime} ; \eta_{1}, \eta_{2}, \ldots, \alpha_{2}\right)\left[2 i \pi / t h^{\prime \prime}\left(\alpha_{2}\right)\right]^{1 / 2} e^{i t h\left(\alpha_{2}\right)+i k \alpha_{2}-i k^{2} /\left(2 t h^{\prime \prime}\left(\alpha_{2}\right)\right)}
\end{gathered}
$$

Integrating now on $\eta_{n-1}$ the two terms of the expression above we obtain the sum of four terms, which are again doubled after the next integration and so on. Lastly the asymptotic expression of $I_{P^{\prime} P^{\prime \prime}}$ become

$$
\frac{1}{2 \pi} \int d k \sum_{i_{1} i_{2} \ldots i_{n}} G\left(P^{\prime}, P^{\prime \prime} ; \alpha_{i_{1}}, \alpha_{i_{2}}, \ldots, \alpha_{i_{n}}\right) \prod_{j=1}^{n} \sqrt{\frac{2 i \pi}{t h^{\prime \prime}\left(\alpha_{i_{j}}\right)}} e^{i t h\left(\alpha_{i_{j}}\right)+i\left[k \alpha_{i_{j}}-\frac{k^{2}}{2 t h^{\prime \prime}\left(\alpha_{i_{j}}\right)}\right]}
$$


In the multiple sum, each of $i_{1}, i_{2}, \ldots, i_{n}$ assumes the value 1 or 2 , corresponding to the two values of the stationary points for each relative momentum. Finally, performing the integration on $k$, we obtain:

$$
\left(\frac{2 \pi}{t}\right)^{\frac{(n-1)}{2}} \sum_{i_{1} i_{2} \ldots i_{n}}^{\prime} G\left(P^{\prime}, P^{\prime \prime} ; \alpha_{i_{1}}, \alpha_{i_{2}}, \ldots, \alpha_{i_{n}}\right) \frac{e^{\frac{i t\left(\sum_{j=1}^{n} \alpha_{i_{j}}\right)^{2}}{2 \sum_{j=1}^{n} 1 / h^{\prime \prime}\left(\alpha_{i_{j}}\right)}}}{\sqrt{i \sum_{j=1}^{n} 1 / h^{\prime \prime}\left(\alpha_{i_{j}}\right)}} \prod_{j=1}^{n} \frac{e^{i t h\left(\alpha_{i_{j}}\right)}}{\sqrt{-i h^{\prime \prime}\left(\alpha_{i_{j}}\right)}}
$$

where $\sum^{\prime}$ means that the terms for which $\sum_{j=1}^{n} 1 / h^{\prime \prime}\left(\alpha_{i_{j}}\right)=0$ are excluded. Such terms indeed give zero after the integration on $k$. After substitution of $\left|\sqrt{i \sum_{j=1}^{n} 1 / h^{\prime \prime}\left(\alpha_{i_{j}}\right)}\right|$ with the lower possible value $\sqrt{|1 /(2 w)|}$, we have asymptotically

$$
\left|\rho_{P^{\prime} P^{\prime \prime}}\right| \leq\left|\frac{2 \pi}{t}\right|^{(n-1) / 2}|2 w|^{-(n-1) / 2} \sum_{i_{1} i_{2} \ldots i_{n}}^{\prime}\left|G\left(P^{\prime}, P^{\prime \prime} ; \alpha_{i_{1}}, \alpha_{i_{2}}, \ldots, \alpha_{i_{n}}\right)\right| .
$$

For the primed sum we may also write:

$$
\begin{aligned}
& \sum_{i_{1} i_{2} \ldots i_{n}}^{\prime}\left|G\left(P^{\prime}, P^{\prime \prime} ; \alpha_{i_{1}}, \ldots, \alpha_{i_{n}}\right)\right| \leq \\
& 2^{n}\left[\sum_{i_{1} i_{2} \ldots i_{n}}^{\prime}\left|\Phi\left(P^{\prime}, \alpha_{i_{1}}, \ldots, \alpha_{i_{n}}\right)\right|\left|\Phi^{*}\left(P^{\prime \prime}, \alpha_{i_{1}}, \ldots, \alpha_{i_{n}}\right)\right|\right] / p
\end{aligned}
$$

where $p$ is the number of terms which contribute with non zero value to the sum. We used the definition of $G\left(P^{\prime}, P^{\prime \prime} ; \eta_{1}, \eta_{2}, \ldots, \eta_{n}\right)$ already given and the fact that $p$ is equal or less than the $2^{n}$ possible combinations of the indexes $i_{j}$.

At this point we will compare the off-diagonal matrix elements with the diagonal ones. Since the last are constant of motion, we do not expect it would change the result, but to obtain a dimensionless expression. To this end we need to establish a further bound. Looking at the definition of the reduced matrix elements we may state that

$$
\left|\rho_{P P}\right|=I_{P P}=\int \Phi\left(P, \eta_{1}, \ldots, \eta_{n}\right) \Phi^{*}\left(P, \eta_{1}, \ldots, \eta_{n}\right) d S
$$

is evidently greater or equal to the result of the integration over a finite volume as the integrand is a positive quantity. Let $-M c \leq \eta_{i} \leq M c$ be a finite integration interval for each variable. Recalling that only $n-1$ of them are independent, as $\eta_{n}=-\sum_{j=1}^{n-1} \eta_{j}$ :

$$
\left|\rho_{P P}\right| \geq(2 M c)^{n-1}\left|\Phi\left(P, u_{1}, u_{2} \ldots\right)\right|^{2}
$$


where $u_{1}, u_{2} \ldots$ denotes an internal point of the hypercube chosen as integration domain. We recall that the function $\left|\Phi\left(P, u_{1}, u_{2} \ldots\right)\right|$ is assumed as bounded everywhere. Comparing now the off-diagonal elements to the diagonal ones we will consider, to be democratic, the ratio of $\left|\rho_{P^{\prime} P^{\prime \prime}}\right|$ to $\left(\left|\rho_{P^{\prime} P^{\prime}}\right|\left|\rho_{P^{\prime \prime} P^{\prime \prime}}\right|\right)^{1 / 2}$. Using the inequalities already found:

$$
\left|\rho_{P^{\prime} P^{\prime \prime}}\right| /\left|\left(\rho_{P^{\prime} P^{\prime}}|| \rho_{P^{\prime \prime} P^{\prime \prime}} \mid\right)^{1 / 2} \leq 2 A\right| t /\left.\tau\right|^{-(n-1) / 2}
$$

The constant $A$ denotes the quantity:

$$
\frac{\left[\sum_{i_{1} i_{2} \ldots i_{n}}^{\prime}\left|\Phi\left(P^{\prime}, \alpha_{i_{1}}, \ldots, \alpha_{i_{n}}\right)\right|\left|\Phi\left(P^{\prime \prime}, \alpha_{i_{1}}, \ldots, \alpha_{i_{n}}\right)\right|\right] / p}{\left|\Phi\left(P^{\prime}, u_{1}^{\prime}, u_{2}^{\prime} \ldots\right)\right|\left|\Phi\left(P^{\prime \prime}, u_{1}^{\prime \prime}, u_{2}^{\prime \prime} \ldots\right)\right|}
$$

and $\tau=\frac{4 \pi \hbar m}{3\left|P^{\prime 2}-P^{\prime \prime 2}\right|}$ is a characteristic time scale of the process such that when $t \gg \tau$ the absolute values of the off diagonal elements are vanishing. It would be pointed out that actually we have decoherence in the basis of (nonrelativistic) kinetic energy $E=\frac{P^{2}}{2 M}$ of the c.m.. Introducing $E$ as a more appropriate variable the decoherence time is better written as $\tau=\frac{2 \pi \hbar m}{3 M\left|E^{\prime}-E^{\prime \prime}\right|}$. It is inversely proportional to the difference $\left|E^{\prime}-E^{\prime \prime}\right|$ and also decreases with increasing total mass $M$ as one may expect intuitively. With $\left|E^{\prime}-E^{\prime \prime}\right|$ of the order of a few $\mathrm{eV}$ and with, say, ten particles, the characteristic time is very small, about $10^{-16}$ sec.

As we anticipated and contrary to our initial prediction, the result we have just found shows that the reduced density matrix diagonalizes on the basis of $\widehat{P}^{2}$ and not on that of total momentum $\widehat{P}$. That is, they are the matrix elements for which $\left|P^{\prime}\right| \neq\left|P^{\prime \prime}\right|$, which we found go to zero as time increases. But our expression for the asymptotic behaviour is not valid if $P^{\prime}=-P^{\prime \prime}$. In such a case, in fact, the function $h(\eta)$ depends only on $\eta^{3}$, we have only a stationary point of the second order and the asymptotic evaluation of our integral has to be worked out separately. To this end, the integration interval in $\eta_{i}$ is split into $(0, \infty)$ and $(-\infty, 0)$ and each of the two integrals is integrated by parts a number of times, differentiating $G\left(P^{\prime}, P^{\prime \prime} ; \eta_{1}, \eta_{2}, \ldots, \eta_{n}\right)$ and integrating the remaining factor of the integrand. Taking only the dominant term into account, asymptotically we have:

$$
\left|\rho_{P^{\prime} P^{\prime \prime}}\right| /\left(\left|\rho_{P^{\prime} P^{\prime}}\right|\left|\rho_{P^{\prime \prime} P^{\prime \prime}}\right|\right)^{1 / 2} \leq B\left|t / \tau^{\prime}\right|^{-(n-1) / 3}\left|\int_{-\infty}^{+\infty} d \beta\left[A_{i}(\beta)\right]^{n}\right|
$$


The constant $B$ is:

$$
\frac{\left|\Phi\left(P^{\prime}, 0,0,0, \ldots, 0\right)\right|\left|\Phi\left(P^{\prime \prime}, 0,0,0,0, \ldots, 0\right)\right|}{\left|\Phi\left(P^{\prime}, u_{1}^{\prime}, u_{2}^{\prime} \ldots\right)\right|\left|\Phi\left(P^{\prime \prime}, u_{1}^{\prime \prime}, u_{2}^{\prime \prime} \ldots\right)\right|}
$$

and $\tau^{\prime}=\frac{2 \pi^{3} \hbar}{3 n^{2}\left|P^{\prime}-P^{\prime \prime}\right| c}$. For values of $\left|P^{\prime}-P^{\prime \prime}\right|$ of a few $e V / c$ and always assuming $n \sim 10$, still $\tau^{\prime} \sim 10^{-16}$ sec. The function $A i(\beta)$ indicates the Airy function $\frac{1}{\pi} \int_{0}^{+\infty} \cos \left(\beta y+y^{3} / 3\right) d y$. We assumed time to be positive and $P^{\prime}>P^{\prime \prime}$. The asymptotic estimate of the other half of the off-diagonal matrix elements for which $P^{\prime}<P^{\prime \prime}$ is immediately obtained since $\left|\rho_{P^{\prime \prime} P^{\prime}}\right|=\left|\rho_{P^{\prime} P^{\prime \prime}}\right|$ by hermiticity. $A i(\beta)$ for large and positive values of $\beta$ tends exponentially to zero. It is also going to zero for $\beta$ which tends towards $-\infty$, but now the behaviour is like $|\beta|^{-1 / 4}$. However, for a sufficient number of particles (five or more) $[A i(\beta)]^{n}$ is integrable. Summing up, $\left|\rho_{P^{\prime} P^{\prime \prime}}\right| /\left(\left|\rho_{P^{\prime} P^{\prime}}\right|\left|\rho_{P^{\prime \prime} P^{\prime \prime}}\right|\right)^{1 / 2}$, if the number of particle is as indicated, also tends asymptotically to zero when $P^{\prime}=-P^{\prime \prime}$ and we have a complete diagonalization on $\widehat{P}$ basis.

These findings are strongly dependent on the assumption that the system of particles is isolated. It presumes a not very realistic situation since from physical viewpoint it is hard to exclude external influences, and even under the slight perturbation the time behaviour we obtained for the reduced matrix elements would not persist. So, even if we intended to present our findings strictly as a mathematical result, a brief discussion about the persistence of the present decoherence effect in the case of external perturbations imposes. We will discuss this point in comparison with the case of a free non-relativistic particle.

The time dependence in the momentum basis of a non-relativistic free particle density matrix elements is simply:

$$
\psi\left(k^{\prime}\right) \psi^{*}\left(k^{\prime \prime}\right) e^{-i t \frac{\left(k^{\prime 2}-k^{\prime \prime 2}\right)}{2 m \hbar}}
$$

where $\psi(k)$ is the initial state. Under a time average the expression above, compared to the diagonal elements, which are constants of motion, becomes:

$$
\frac{\varrho_{k^{\prime} k^{\prime \prime}}}{\varrho_{k^{\prime} k^{\prime}}^{1 / 2} \varrho_{k^{\prime \prime} k^{\prime \prime}}^{1 / 2}}=\frac{\psi\left(k^{\prime}\right) \psi^{*}\left(k^{\prime \prime}\right)}{\left|\psi\left(k^{\prime}\right)\right|\left|\psi\left(k^{\prime \prime}\right)\right|} \frac{1}{t} \int_{0}^{t} e^{-i t / \tau_{\text {free }}} d t
$$

with, denoting as $T_{k}$ the kinetic energy, a "decoherence time" $\tau_{\text {free }}=\frac{\hbar}{\left|T_{k^{\prime}}-T_{k^{\prime \prime}}\right|}$. Since the integral gives a limited oscillating term the asymptotic behaviour 
for the correlations of two different eigenstates of kinetic energie is as the inverse power of time, namely as $\left(t / \tau_{\text {free }}\right)^{-1}$. Let now consider a constant perturbation given as a generic function of the energy $\varepsilon f(T)$, which commutes with the free Hamiltonian, and defines $T_{k^{\prime \prime}}=T_{k^{\prime}}-\Delta$.

Taking the time average requires to substitute the former integral with the new expression:

$$
\frac{1}{t} \int_{0}^{t} d t e^{-\frac{i t}{\hbar}\left[\Delta+\varepsilon f\left(T_{k^{\prime}}\right)-\varepsilon f\left(T_{k^{\prime}}-\Delta\right)\right]}
$$

For each value of $\Delta$ for which the argument of the exponential is zero the integral gives just one. Then for each $k^{\prime}$ there are so many values of $k^{\prime \prime}$ as the zeroes of the function $f\left(T_{k^{\prime}}\right)-f\left(T_{k^{\prime}}-\Delta\right)$ for which the averaged matrix elements do not go to zero with increasing time. In the case of decoherence we considered the role of a free particle is played by the centre of mass. Adding any constant perturbation $\varepsilon f\left(T_{P}\right)$ to the Hamiltonian of our isolated system will not disturb at all the asymptotic behaviour of any reduced matrix element. In fact we merely have a change of their phases, whereas their absolute values are left unchanged. This is a rather artificial consideration, since the sought disturbance is not very realistic. However this example may deserve to show that our case of decoherence is persistent under disturbances which only touches the matrix elements phases.

Coming back to the case of the free non-relativistic particle, we now consider a perturbation due to a constant force, adding to the free Hamiltonian the term $H^{\prime}=x F$, which may be written as $x m c / \tau_{l}$. Choosing the acting force as the weight force, the quantity $\tau_{l}$ is about $3 \times 10^{7} \mathrm{sec}$.

It is easy to see that the wave function of the free falling particle evolves with time in momentum basis as:

$$
\psi\left(k+m c t / \tau_{l}\right) e^{-\frac{i}{2 m \hbar}\left(k^{2} t+k \frac{m c}{\tau_{l}} t^{2}+\frac{m^{2} c^{2}}{3 \tau_{l}^{2}} t^{3}\right)}
$$

with the same initial condition as before. The above expression means that in addition to the phase variation with time of each plane wave component, the wave packet "moves" in momentum space at the rate of $m c / \tau_{l}$ per sec. This entails that, for a square integrable wave function, $\psi\left(k+m c t / \tau_{l}\right)$ goes in the long run to zero for any fixed value of $k$ and hence any density matrix element with fixed indexes do the same. For a wave packet with initial spread $\Delta k$ equal to some fractions of $m c$ it will happen after a time of the order of 
year. So we may disregard this kind of time dependence for the first minutes. Within this approximation the time average of the normalized density matrix elements gives:

$$
\frac{\psi\left(k^{\prime}\right) \psi^{*}\left(k^{\prime \prime}\right)}{\left|\psi\left(k^{\prime}\right)\right|\left|\psi\left(k^{\prime \prime}\right)\right|} \frac{1}{t} \int_{0}^{t} e^{-\frac{i t}{2 m \hbar}\left(k^{\prime 2}-k^{\prime \prime 2}\right)-i \frac{m c\left(k^{\prime}-k^{\prime \prime}\right)}{2 m \hbar \tau_{l}} t^{2}} d t
$$

We rewrite the time factor as

$$
\frac{1}{t} \int_{0}^{t} e^{-i\left(\frac{t}{\tau_{\text {free }}}+\frac{t^{2}}{\tau_{m}^{2}}\right)} d t
$$

where $\tau_{\text {free }}=\frac{\hbar}{\left|T_{k^{\prime}}-T_{k^{\prime \prime}}\right|}$ was already encountered and $\tau_{m}=\frac{\left|k^{\prime}+k^{\prime \prime}\right|}{m c}\left(\tau_{\text {free }} \tau_{l}\right)^{1 / 2}$. We point out that $\tau_{m}$ is generally greater than $\tau_{\text {free }}$. The asymptotic expansion gives:

$$
\frac{1}{t} \int_{0}^{t} e^{-i\left(\frac{t}{\tau_{\text {free }}}+\frac{t^{2}}{\tau_{m}^{2}}\right)} d t \simeq \frac{e^{-i t / \tau_{\text {free }}}}{t} \int_{0}^{t} e^{-i \frac{t^{2}}{\tau_{m}^{2}}} d t+\cdots
$$

The first dominating term is easy written with a couple of Fresnel integrals $C(z)$ and $S(z)$ where $z=\sqrt{2 / \pi} t / \tau_{m}$ :

$$
\frac{e^{-i t / \tau_{\text {free }}}}{t} \int_{0}^{t} e^{-i \frac{t^{2}}{\tau_{m}^{2}}} d t=\frac{\sqrt{2 / \pi} e^{-i t / \tau_{\text {free }}}}{t / \tau_{m}}\left[C\left(\sqrt{2 / \pi} t / \tau_{m}\right)-i S\left(\sqrt{2 / \pi} t / \tau_{m}\right)\right]
$$

For $z<1$ the Fresnel integrals are increasing functions of the argument, and both of them goes to the constant value $1 / 2$ in the limit of large $z$. In conclusion in the case of non-relativistic particle subjected to weight force we have still decoherence with a $1 / t$ asymptotic behaviour. The decoherence time however is now the greater quantity $\tau_{m}$ instead of $\tau_{\text {free }}$. We also examined our system of free quasi-relativistic particles, in the case $\left|P^{\prime}\right| \neq\left|P^{\prime \prime}\right|$, under the perturbation of the weight force applied to the c.m.. We encountered again three characteristic time scales, one of which is the already defined $\tau$. At time $t \gg \tau$ but shorter than the analogue of $\tau_{m}$ and in the worse situation the off-diagonal density matrix elements differ from zero. We have still decoherence, but with a greater decoherence time, the analogous of $\tau_{m}$. However the time behaviour for the decays of correlations is now as $1 / t^{n-1}$.

We may conclude that the decoherence mechanism we presented here is as robust or a little more robust to perturbations than the non-relativistic free particle case, i. e. not very robust. 
For the sake of completeness, we will also consider the case of relativistic particles, using the so-called "relativistic Schroedinger equation". This equation has been used in various situations, for example to study the stability of matter constituted of fixed nuclei and electrons and the collapse of stars of self-gravitating bosons or fermions 20. It is obtained replacing in the usual Schroedinger equation the kinetic energy of a particle with its full relativistic expression. Let us suppose that there is a Lorentz frame such that $\sqrt{\left\langle\eta_{i}^{2}\right\rangle_{P}} \ll|P / n|$ for a set $R$ of values of $P$, the average value in question being calculated by means of the above mentioned function $\Phi\left(P, \eta_{1}, \eta_{2}, \ldots, \eta_{n}\right)$. In these conditions we can use a procedure similar to the previous one to calculate the time behaviour of the off-diagonal reduced density matrix elements whose indexes $P^{\prime}, P^{\prime \prime}$ refer to eigenvalues of the total impulse belonging to the set $R$. In fact, the kinetic energy $\sqrt{m^{2} c^{4}+\left(P / n+\eta_{i}\right)^{2}}$ of each particle $i$ can be developed with good approximation in power of $\eta_{i}$ retaining the terms up to $\eta_{i}^{3}$. Repeating, mutatis mutanda, the previous steps we once again find that the off-diagonal elements of the reduced density matrix compared to diagonal ones decrease asymptotically with time, as follows:

$$
\left|\rho_{P^{\prime} P^{\prime \prime}}\right| /\left(\left|\rho_{P^{\prime} P^{\prime}}\right|\left|\rho_{P^{\prime \prime} P^{\prime \prime}}\right|\right)^{1 / 2} \leq 2 C\left|t / \tau_{r}\right|^{-(n-1) / 2} \quad .
$$

The constant $C$ is:

$$
\frac{\left[\sum_{i_{1} i_{2} \ldots i_{n}}^{\prime}\left|\Phi\left(P^{\prime}, \gamma_{i_{1}}, \gamma_{i_{2}}, \ldots\right)\right|\left|\Phi^{*}\left(P^{\prime \prime}, \gamma_{i_{1}}, \gamma_{i_{2}}, \ldots\right)\right|\right] / p}{\left(\left|\Phi\left(P^{\prime}, v_{1}^{\prime}, v_{2}^{\prime} \ldots\right)\right|\left|\Phi\left(P^{\prime \prime}, v_{1}^{\prime \prime}, v_{2}^{\prime \prime} \ldots\right)\right|\right)^{1 / 2}}
$$

denoting as $\gamma_{1}, \gamma_{2}$ the two new stationary points. The decoherence time is now

$$
\tau_{r}=\frac{\pi \hbar E^{\prime 3} E^{\prime \prime 3}}{n M^{4} c^{8}\left|E^{\prime \prime}-E^{\prime \prime}\right|\left(E^{\prime \prime 2}+E^{\prime} E^{\prime \prime}+E^{\prime \prime 2}\right)}
$$

where $E^{2}=M^{2} c^{4}+P^{2} c^{2}$. For $E^{\prime} \sim E^{\prime \prime} \sim 10 M c^{2},\left|E^{\prime}-E^{\prime \prime}\right| \sim 1 e V$ and considering about ten particles, we obtain $\tau_{r} \sim 10^{-13}$ sec. All of this applies, we remind, if both $\left|P^{\prime}\right|$ and $\left|P^{\prime \prime}\right|$ belong to $R$. As before, the result is that the reduced matrix diagonalizes on the basis of $\widehat{P}^{2}$. But here too our expression for the asymptotic behaviour is not valid if $\left|P^{\prime}\right|=\left|P^{\prime \prime}\right|$. In that case we have a double stationary point, the evaluation of the off diagonal reduced matrix elements has to be worked out separately with the result that asymptotically $\left|\rho_{P^{\prime} P^{\prime \prime}}\right|$ depends on time as $|t|^{-(n-1) / 3}$. 


\section{CONCLUDING REMARKS}

Using non-relativistic quantum mechanics, and within a reference frame in which the system is on average at rest (i.e. its average total momentum is zero), we have shown that a one-dimensional system of a sufficient number of free quasi-relativistic particles, even if isolated, may decohere on the basis of the total momentum if we considers correction to kinetic energy to terms of second order in $1 / c$. In other words, we have established, under the conditions stated above, a superselection rule for the total momentum. For the sake of completeness, we obtained similar results using the full relativistic expression for the kinetic operator for those Lorentz frames in which the relative momenta are much smaller than $P / n$, where $P$ is the total momentum. The decoherence we are talking about was obtained tracing upon the relative momenta. Our choice of that coarse-graining seems natural if we want to look at the system as a whole. The higher the number of particles, the faster the decoherence, meaning that it becomes more effective in the macroscopic limit. What we found in the case of relativistic corrections within a non-relativistic quantum mechanics framework may reasonably suggest that isolated systems of charged or gravitational interacting particles, for which the approximate Hamiltonian we mentioned is adopted, present decoherence on the basis of total momentum if we look at the system as a whole and ignore the internal degrees of freedom. Systems of this sort might include atoms with many electrons, for instance. True, the Hamiltonian in question is not only approximated but also ignores spin, so it may be considered "semi-classic". The presumed effect is interesting anyway. If the number of particles is very big, such that the system may be considered macroscopic, it cannot be supposed to be isolated, due to the extraordinary density of its energy levels and to the action of surrounding objects, even if this tends towards zero. Also we learned that our decoherence mechanism is not bery robust to perturbations and so other non relativistic interaction terms will cause a more effective and different phenomenologically decoherence of the c.m. variables. The action of the external environment will certainly prevail, so that the reduced density matrix for the collective variable of our system will diagonalize on the basis of the position, as shown in many examples 11. But a mesoscopic object, with a very small number of components, may probably be considered isolated, if only for a short period of time. In which 
case we get the following situation: macroscopic bodies appear in a localized state, while particles made up of a small number of components would appear in well defined states of the total momentum. Further, while not being realistic, the model we have discussed for fully relativistic particles can be applied to the case of multiple production in the two bodies collision at high energy. As it is well known, the secondary particles produced in these experiments have a transverse momentum $p_{t}$, which is negligible compared with the momentum parallel to the collision axis $\left(p_{l}\right)$, and in fact, one-dimensional variables have been introduced already to describe the process 21. A very popular phenomenological model considers the secondaries in the final state as non-interacting particles grouped in clusters having a classical statistical distribution in the longitudinal momentum or rapidity[22]. Let us think of a cluster in terms of our model (adapted for the purpose) and consider the relative momenta of the secondaries emitted from it to have values $\sim p_{t}$, which is much less (outside the central zone) than the total momentum of the system. Then we must conclude that the reduced density matrix of clusters rapidly diagonalizes on the basis of their total momentum. Which justifies their description in terms of classical statistical distribution.

\section{References}

[1] W. H. Zurek, Phys. Rev. D 26 (1982) 1862; E. Joos and H. D. Zeh, Zeits. Phys. B 59 (1985) 223; A. O. Caldeira and A. J. Legget, Phys. Rev. A 31 (1985) 1059; W. H. Zurek, Phys. Today 44 (1991) 36, ibid 46 (1993) 81; W. H. Zurek, Prog. Theor. Phys. 89 (1993) 281; J. P. Paz and W. H. Zurek, Phys. Rev. D 48 (1993) 2728; R. Omnes, The Interpretation of Quantum Mechanics Princeton Un. Press (1994), chap. 7.

[2] W. H. Zurek, Phys. Rev. D 24 (1981) 1516; W. H. Zurek, Phys. Rev. D 26 (1982) 1862; A. Albrecht, Phys. Rev. D 46 (1992) 5504; A. Albrecht, Phys. Rev. D 48 (1993) 3768.

[3] E. Joos and H. D. Zeh, Zeits. Phys. B 59 (1985) 223; A. O. Caldeira and A. J. Legget, Physica A 121 (1983) 587; W. G. Unruh, W. H. Zurek, 
Phys. Rev. D 40 (1989) 1071; J. P. Paz, S. Habib, W. H. Zurek Phys. Rev. 47 (1993) 488.

[4] M. R. Gallis and G. N. Fleming, Phys. Rev. A 42 (1989) 38.

[5] M. Tegmark, Found. Phys. Lett. 6 (1993) 571.

[6] S. W. Hawking, Comm. Math. Phys. 87 (1982) 395.

[7] J. Ellis, S. Mohanty and D. V. Nanopoulos, Phys. Lett. B 221 (1989) 113.

[8] J. J. Halliwell, Phys. Rev. D 58 (1998) 105015-1.

[9] B. Carazza, Found. Phys. Lett. 10 (1997) 581; J. C. Flores quantph/9803032 (1998).

[10] A. O. Caldeira and A. J. Legget, Phys. Rev. A 31 (1985) 1059.

[11] E. Joos and H. D. Zeh, Zeits. Phys. B 59 (1985) 223.

[12] R. Omnes, Phys. Rev. A 56 (1997) 3383.

[13] N. Gisin and I. C. Percival, J. Phys. A 25 (1992) 5677; R. Scheck, T. Brun and I. C. Percival, J. Phys. A 28 (1995) 5041.

[14] E. Calzetta and B. L. Hu, hep-th/9501040 (1998).

[15] C. Anastopoulos, Phys. Rev. D 54 (1996) 1600.

[16] L. Landau and E. Lifshitz, The Classical Theory of Fields AddisonWesley, Cambridge (1951), chap. 8.

[17] J. Percival, J. Phys. A 27 (1994) 1003.

[18] W. H. Zurek, Phys. Rev. D 24 (1981) 1516.

[19] A. Erdely, Asymptotic Expansions Dover Publications, N.Y. (1956), chap. 2. 
[20] J. M. Levy-Leblond, J. Math. Phys. 10 (1969) 806; I. Daubechies and E. H. Lieb, Comm. Math. Phys. 90 (1983) 497; A. Martin, Phys. Lett. B 214 (1988) 561; J. L. Basdevant, A. Martin and J. M. Richard, Nucl. Phys. B 343 (1990) 60; B. Carazza and P. Quarati, Nuovo Cimento 106 A (1993) 1353.

[21] R. Feynman, Phys. Rev. Lett. 23 (1969) 1415.

[22] M. Anselmino, A. Ballestrero and E. Predazzi, Nuovo Cimento 36 A (1976) 205; M. Anselmino, A. Ballestrero, B. Carazza and E. Predazzi, Nuovo Cimento 43 A (1978) 152; G. Giacomelli and M. Jacob, Phys. Rep. 55 (1979) 1. 